\title{
Veil-of-Ignorance Reasoning Favors the Greater Good
}

\author{
Karen Huang ${ }^{\mathrm{a}, \mathrm{b}} *$ \\ Joshua D. Greene \\ Max Bazerman ${ }^{\mathrm{b}}$ \\ ${ }^{a}$ Department of Psychology, Harvard University, Cambridge, MA 02138 \\ ${ }^{b}$ Harvard Business School, Harvard University, Boston, MA 02163 \\ *Correspondence should be addressed to: \\ Karen Huang (karenhuang@g.harvard.edu)
}

\section{Acknowledgments}

Thanks to F. Cushman, D. Gilbert, A.W. Brooks, N. Barak-Corren, I. Gabriel, and members of the Cushman and Greene labs for comments; and S. Worthington for statistical support. This research was supported by the Future of Life Institute (\#2015-146757) and Harvard Business School. 


\begin{abstract}
The "veil of ignorance" is a moral reasoning device designed to promote impartial decision-making by denying decision-makers access to potentially biasing information about who will benefit most or least from the available options. Veil-of-ignorance reasoning was originally applied by philosophers and economists to foundational questions concerning the overall organization of society. Here we apply veil-of-ignorance reasoning in a more focused way to specific moral dilemmas, all of which involve a tension between the greater good and competing moral concerns. Across six experiments $(N=5,785)$, three pre-registered, we find that veil-of-ignorance reasoning favors the greater good. Participants first engaged in veil-ofignorance reasoning about a specific dilemma, asking themselves what they would want if they did not know who among those affected they would be. Participants then responded to a more conventional version of the same dilemma with a moral judgment, a policy preference, or an economic choice. Participants who first engaged in veil-of-ignorance reasoning subsequently made more utilitarian choices in response to a classic philosophical dilemma, a medical dilemma, a real donation decision between a more vs. less effective charity, and a policy decision concerning the social dilemma of autonomous vehicles. These effects depend on the impartial thinking induced by veil-of-ignorance reasoning and cannot be explained by a simple anchoring account, probabilistic reasoning, or generic perspective-taking. These studies indicate that veilof-ignorance reasoning may be a useful tool for decision-makers who wish to make more impartial and/or socially beneficial choices.
\end{abstract}

Keywords: ethics, decision-making, policy-making, procedural justice, fairness 


\section{Veil-of-Ignorance Reasoning Favors the Greater Good}

The philosopher John Rawls proposed a famous thought experiment, aimed at identifying the governing principles of a just society (1). Rawls imagined decision-makers who've been denied all knowledge of their personal circumstances. They don't know whether they, as individuals, are rich or poor, healthy or ill, or in possession of special talents or abilities. Nor do they know the social groups to which they belong, as defined by race, class, gender, etc. The decision-makers are assumed to be purely self-interested, but their decisions are constrained by the absence of information that they could use to select principles favorable to their personal circumstances. Rawls referred to this epistemically restricted state as being behind a "veil of ignorance".

Rawls conceived of this hypothetical decision as a device for helping people in the real world think more clearly and impartially about the organizing principles of society. A just social order, he argued, is one that selfish people would choose if they were constrained to choose impartially, in the absence of potentially biasing information. Some empirical researchers have adapted Rawls' thought experiment to the lab, asking ordinary people to evaluate candidate organizing principles by engaging in veil-of-ignorance reasoning (2). Here, we depart from the conventional use of the veil of ignorance as a device for thinking about the general organization of society. Instead, we apply veil-of-ignorance reasoning to a set of more specific moral and social dilemmas. These dilemmas, though more restricted in scope than Rawls' foundational dilemma, are nevertheless of broad social significance, with life-and-death consequences in the domains of healthcare, international aid, and automated transportation. What effect, if any, does veil-of-ignorance reasoning have on people's responses to such dilemmas? 
We predict that veil-of-ignorance reasoning will cause people to make more utilitarian judgments, by which we mean judgments that maximize collective welfare. ${ }^{*}$ This result is by no means guaranteed, as there are reasons to think that veil-of-ignorance reasoning could have the opposite effect, or no effect at all. Rawls was one of utilitarianism's leading critics (1), suggesting that veil-of-ignorance reasoning might reduce utilitarian judgment. And even if veilof-ignorance reasoning were to support utilitarian choices, it's possible that people's ordinary responses to moral dilemmas implicitly reflect the lessons of veil-of-ignorance reasoning, such that engaging in explicit veil-of-ignorance reasoning would have no additional effect.

Despite Rawls' renown as a critic of utilitarianism, our predicted results are not necessarily incompatible with Rawls' philosophy, as the dilemmas employed here are not designed to distinguish between a utilitarian decision principle and Rawls" "maximin" principle $(1,3) .^{\dagger}$ Our predictions are, however, most closely aligned with the ideas of Rawls’

\footnotetext{
* Following convention in the psychology and cognitive neuroscience literatures, we refer to these judgments as "utilitarian", but one could also call them "consequentialist", a label that does not assume that saving more lives necessarily implies greater overall happiness. In addition, in calling these judgments "utilitarian" we are not claiming that the people who make them are in any general way committed to utilitarianism (14).

† Rawls" "maximin" principle favors whatever outcome maximizes the welfare of the least welloff person. For example, in the footbridge case, the least well-off people under each option experiences equally bad outcomes, namely death by trolley. Thus, one might expect a Rawlsian to be indifferent between the two options or to favor the utilitarian option, invoking the utilitarian principle as a secondary consideration. With that said, the judgment that it's wrong to push the
} 
contemporary and critic, John Harsanyi, an influential economist who independently conceived of veil-of-ignorance reasoning and argued that it provides a decision-theoretic foundation for a utilitarian social philosophy (4-5).

To illustrate our application of veil-of-ignorance reasoning, consider the well-known footbridge dilemma, in which one can save five people in the path of a runaway trolley by pushing a person off of a footbridge and into the trolley's path (6). The utilitarian option is to push, as this maximizes the number of lives saved, but relatively few people favor the utilitarian option in this case, a result of negative affective responses to this actively, directly, and intentionally harmful action (7-8).

What effect might veil-of-ignorance reasoning have on a case such as this? Following Hare (9), you might imagine that you are going to be one of the six people affected by the footbridge decision (one of the five on the tracks or the one who could be pushed). You might assume that you have even odds of being any one of them. (We note that Rawls' version of the veil of ignorance assumes unknown odds rather than even odds. ${ }^{\ddagger}$ Would you, from a purely self-

man off the footbridge may be defended in broadly Rawlsian terms, appealing to the idea that, “each person possesses an inviolability founded on justice that even the welfare of society as a whole cannot override" (1, p. 3). See, for example, Sandel (30, Chapter 2).

$\$$ In Rawls’ version of the veil of ignorance, the decision makers assume that their odds of occupying any particular position in society are unknown. Following Harsanyi, we make an "equi-probability" assumption, instructing subjects that they have even odds of being each of the people affected by the decision. In other words, we make the decision a matter of "risk", rather than "ambiguity" (31). We do this for two related reasons. First, it is not our purpose to examine 
interested perspective, want the decision-maker to push, giving you a 5 out of 6 chance of living? Or would you want the decision-maker to not push, giving you a 1 out 6 chance of living? Here, we suspect (and confirm in Study 1) that most people prefer that the decision-maker push, increasing one's odds of living.

But, what, if anything, does this imply about the ethics of the original moral dilemma? As noted above, most people say that it would be wrong to push the man off the footbridge. And yet a Rawlsian (or Harsanyian) argument seems to imply that pushing is the fairer and more just option: It's what those affected by the decision would want if they didn't know which positions they would occupy. In the studies presented here, we follow a two-stage procedure, as suggested by the foregoing argument. First, participants consider a veil-of-ignorance version of a moral dilemma, reporting on what they would want a decision-maker to do if they did not know who they would be among those affected by the decision. Second, participants respond to a standard

the effect of Rawls' specific version of veil-of-ignorance reasoning, but rather to determine whether some kind of veil-of-ignorance reasoning, embodying a principle of impartiality, can influence moral judgment and, more specifically, induce people to make choices that more reliably favor the greater good. Second, and more positively, we believe that Rawls' assumption of unknown odds, rather than even odds, makes little sense given the rationale behind the veil-ofignorance thought experiment: If the function of the veil is to constrain the decision-makers into perfect impartiality, then why not give the interests of each person exactly equal weight by giving the decision-makers exactly even odds of being each person? We know of no compelling justification for assuming that the odds are anything other than exactly equal. (See 12, p. 383$385)$. 
version of the same dilemma, reporting on the moral acceptability of the proposed action. (In Study 3 participants in the second stage make a real-stakes choice instead of a hypothetical judgment.) The question, then, is whether engaging in veil-of-ignorance reasoning in the first stage influences ordinary moral judgment in the second stage. To be clear, we are not comparing the responses in the veil-of-ignorance exercise with responses to the standard moral dilemma. Instead, we investigate the influence of veil-of-ignorance reasoning, induced through the veil-ofignorance exercise, on responses to the standard moral dilemma.

In applying veil-of-ignorance reasoning, we are not only attempting to influence moral judgment, but to do so through a kind of reasoning. By this, we mean that the influence occurs through a conscious valuation process that is constrained by a need for consistency-either with a general principle, with related judgments, or both (10). We expect that, in the second stage, participants will explicitly consider the normative relationship between the moral judgment they are currently making and the judgment that they made in the first stage, a self-interested decision from behind a veil of ignorance. Moreover, we expect that they will be inclined to make their current moral judgment consistent with their prior veil-of-ignorance judgment. In other words, we predict that participants will think something like this: "If I didn't know which of the six people I was going to be, I would want the decision-maker to push. But when I think about pushing, it feels wrong. Nevertheless, if pushing is what I'd want from an impartial perspective, not knowing who I was going to be, then perhaps it really is the right thing to do, even if it feels wrong."

Thus, through this procedure, we encourage participants to absorb the philosophical insight of the veil-of-ignorance thought experiment and apply this idea in their subsequent judgments. The veil-of-ignorance exercise is intended to influence judgment through conscious 
and explicit reasoning. This effect would be significant, in part, because evidence for moral reasoning beyond simple cost-benefit reasoning (11-15) is rare (16).

Beyond moral psychology, the effects of veil-of-ignorance reasoning may be of practical significance, as people's responses to moral dilemmas are often conflicted and carry significant social costs (12). Consider, for example, the social dilemma of autonomous vehicles (AVs) (17), featuring a tradeoff between the safety of AV passengers and that of others, such as pedestrians. As a Mercedez-Benz executive discovered (18), an AV that prioritizes the safety of its passengers will be criticized for devaluing the lives of others. But a "utilitarian" AV that values all lives equally will be criticized for its willingness to sacrifice its passengers. This paradox is reflected in the judgments of ordinary people, who tend to approve of utilitarian AVs in principle, but disapprove of enforcing utilitarian regulations for AVs (17). Likewise, people may feel conflicted about bioethical policies or charities that maximize good outcomes, with costs to specific, identifiable parties (12). We ask whether the impartial perspective encouraged by veilof-ignorance reasoning can influence people's responses to these and other dilemmas. This research does not assume that such an influence would be desirable. However, to the extent that people value impartial decision procedures or collective well-being, such an influence would be significant.

Across six studies, we investigate the influence of the veil of ignorance on moral judgment. We begin with the footbridge dilemma (Study 1) because it is familiar and well characterized. In subsequent studies we employ cases with more direct application, including a decision with real financial stakes (Study 3). Across all cases, we predict that participants' responses to the veil-of-ignorance versions will tend to be utilitarian, simply because this maximizes their odds of a good outcome. Critically, we expect participants to align their later 
moral judgments with their prior veil-of-ignorance preferences, causing them to make more utilitarian moral judgments, favoring the greater good, as compared to control participants who have not engaged in veil-of-ignorance reasoning.

\section{Experimental Designs and Results}

Study 1 ( $N=264)$ employs the footbridge dilemma: The decision-maker can save five people in the path of a runaway trolley by pushing a person off of a footbridge and into the trolley's path (6). The utilitarian option is to push, as this saves more lives, but relatively few favor this option, largely due to negative affective responses (7-8). Study 1's veil-of-ignorance (VOI) condition employs the two-stage procedure described above. In the VOI version (stage 1), participants imagined having equal odds of being each of the six people affected by the decision: the five people on the tracks and the $6^{\text {th }}$ person who could be pushed. Participants were asked whether they would want the decision-maker to push, giving the participant a 5 out of 6 chance of living, or not push, giving the participant a 1 out of 6 chance of living. Here (and in all subsequent studies) most participants gave utilitarian responses to the VOI version of the dilemma. In stage 2 of the VOI condition, participants responded to the standard footbridge dilemma as the decision-maker, evaluating the moral acceptability of pushing, using a dichotomous response and a scale rating. In the control condition there is only one stage, wherein participants respond to the standard dilemma. Critically, the key dependent measures for both conditions were responses to the standard dilemma. In other words, we ask whether first completing the VOI version affects subsequent responses to the standard dilemma.

As predicted, participants in the VOI condition gave more utilitarian responses to the standard footbridge dilemma (38\%, [95\% CI: 30\%, 47\%]), as compared to control participants (24\%, [95\% CI: 18\%, 32\%]; logistic regression, $p=.018)$. Likewise, participants rated the 
utilitarian response as more morally acceptable in the VOI condition $(M=3.32, S D=2.05)$ as compared to the control condition $(M=2.70, S D=1.65)$ (linear regression, $t(262)=2.74, p=$ .007). (Fig. 1A-2A. See Supporting Information for detailed procedures, materials, and results for all studies. See Studies 4-5 for additional control conditions.)

Study $2(N=894)$ employs dilemmas concerning bioethics and the ethics of AVs. In the bioethics case, participants considered taking oxygen away from a single hospital patient to enable the surgeries of 9 incoming earthquake victims. In the VOI version of the bioethical case, participants were asked how they would want the oxygen to be allocated if they knew they had a 1 in 10 chance of being the single patient and a 9 in 10 chance of being one of the 9 earthquake victims (19). In the AV policy case, participants considered whether AVs should be required to minimize the total loss of life (i.e., be utilitarian), for example, saving 9 pedestrians by swerving into a wall, but killing the AV's passenger (17). In the VOI AV case, participants were asked if they would want the AV to swerve into the wall given a 1 in 10 chance of being in the AV and 9 in 10 chance of being one of the 9 pedestrians. As predicted, participants in the VOI condition gave more utilitarian responses to the standard bioethical dilemma (54\%, [95\% CI: 49\%, 59\%], as compared to control $(43 \%,[95 \%$ CI: 39\%, 47\%], $p=.001)$. Likewise, participants in the VOI condition gave more utilitarian responses to the standard AV dilemma (83\%, [95\% CI: 79\%, 87\%], as compared to control (58\%, [95\% CI: 54\%, 62\%], $p<.001)$ (Figs. 1B-C). The rating scale results showed a similar pattern: Participants in the VOI condition reported taking the patient off oxygen as more morally acceptable $(M=4.11, S D=1.90)$ compared to participants in the control condition $(M=3.40, S D=1.75 ; t(892)=5.71, p<.001)$. Similarly, participants in the VOI condition reported swerving as more morally acceptable $(M=5.25, S D=1.74)$ compared to participants in the control condition $(M=4.16, S D=1.83 ; t(892)=8.86, p<.001)$. (Figs. $2 \mathrm{~B}-\mathrm{C}$.) 
Study 3 ( $N=928)$ examines a real-stakes setting: charitable donations. U.S. participants chose to donate $\$ 200$ to one of two real charities (with one randomly selected participant's decision determining the actual outcome). Donating to the more effective/utilitarian charity can be expected to cure two people of blindness in India. Donating to the other charity can be expected to cure one person of blindness in the U.S. In the VOI condition, participants were first asked where they would want the $\$ 200$ to go if they knew they had a 1 in 3 chance of being an American who would be cured by a donation to the U.S. charity and a 2 in 3 chance of being an Indian who would be cured by a donation to the Indian charity. They then made their real donation decisions. As predicted, participants in the VOI condition more often chose to donate to the more effective/utilitarian charity $(62 \%,[95 \%$ CI: 58\%, 67\%]), as compared to control participants, who only made the real donation decision (54\%, [95\% CI: 50\%, 58\%], $p=.0099)$ (Fig. 1D).

We've hypothesized that the effects observed in Studies 1-3 are due to the influence of VOI reasoning itself, inducing a more impartial mindset that promotes concern for the greater good. An alternative explanation is that participants in the VOI condition are simply "anchoring" on their prior utilitarian responses to the VOI versions, giving subsequent utilitarian responses due to a generic tendency toward consistency. (By "anchoring," we mean giving a subsequent response that is the same as a prior response, rather than referring to anchoring in the more specific sense defined by Tversky and Kahneman (20)). Our hypothesis also appeals to a desire for consistency, but we hypothesize that participants are engaging in a specific kind of moral reasoning, mirroring the reasoning of Rawls and Harsanyi, whereby participants perceive a connection between what is morally defensible and what they would want if they did not know whom they would be among those affected by the decision. 
Study 4 ( $N=1,574$; pre-registered) tests this alternative hypothesis while replicating the VOI effect using the AV dilemma. Study 4 employs an additional anchoring control condition in which participants first respond to a standard (non-VOI) dilemma that reliably elicits utilitarian responses. (Deciding whether to destroy a sculpture to save the lives of two people.) As predicted, participants in the VOI condition gave more utilitarian responses $(75 \%,[95 \%$ CI: $70 \%, 79 \%])$, as compared to simple control (50\%, [95\% CI: 46\%, 54\%]; $p<.001)$, and anchoring control (55\%, [95\% CI: 51\%, 60\%]; $p<.001)$. Likewise, participants rated the utilitarian policy as more morally acceptable in the VOI condition $(M=4.91, S D=1.71)$, as compared to those in the simple control condition $(M=3.82, S D=1.86 ; t(1571)=9.65, p<$ $.001)$, and as compared to those in the anchoring control condition $(M=4.10, S D=1.72 ; t(1571)$ $=7.13, p<.001)$. (Figs. 1E, 2D).

Further alternative explanations appeal to features of the VOI dilemma not captured by Study 4's anchoring control condition. More specifically, participants in the VOI condition are asked to engage in numerical reasoning and probabilistic reasoning. This could, perhaps, induce a mindset favoring expected utility calculations, as prescribed by rational choice models of decision-making (21). The VOI condition also asks participants to engage in a limited kind of perspective-taking (22), as participants are asked to consider the effects of the decision on all affected. These and other task features could potentially induce more utilitarian responses to the standard dilemma. These features are essential to VOI reasoning, but a further essential feature of VOI reasoning (as implemented here) is its relation to impartiality, whereby one has an equal probability of being each person affected. Thus, Study 5 ( $N=735$; pre-registered), which again uses the AV dilemma, employs a more stringent control condition in which participants first respond to a modified VOI dilemma in which the probabilities are reversed. That is, one has a 9 
in 10 chance of being the single person in the $\mathrm{AV}$ and a 1 in 10 chance of being one of the 9 pedestrians in the AV's path. Reversing the probabilities disconnects VOI reasoning from impartiality, as one no longer has an equal probability of being each person affected. Because it is the impartiality of VOI reasoning that gives it its moral force, we do not expect this reversedVOI reasoning to have the same effect. As predicted, participants in the VOI condition gave more utilitarian responses $(73 \%,[95 \% \mathrm{CI}: 69 \%, 78 \%])$, as compared to the reversed-VOI control condition (64\%, [95\% CI: 59\%, 69\%]; $p=.006)$. Likewise, participants rated the utilitarian policy as more morally acceptable in the VOI condition $(M=4.89, S D=1.77)$, as compared to those in the reversed-VOI control condition $(M=4.49, S D=1.80 ; t(733)=3.03, p=.003)$ (Figs. 1F, 2E.)

Finally, Study 6 ( $N=1,390$; pre-registered) asks whether VOI reasoning transfers across cases. Participants in the transfer-VOI condition first responded to two VOI cases that are not tightly matched to the AV case, before responding to the standard AV case. Study 6 employed a simple control condition as in Study 1 and a two-dilemma anchoring control condition similar to that of Study 4. Contrary to our predictions, we found no significant differences in participants' responses to the $\mathrm{AV}$ case in the transfer-VOI condition (57\%; [95\% CI: 52\%, 63\%]), as compared to simple control (53\%; [95\% CI: 49\%, 57\%]; $p=.249$ ), and anchoring control (54\%; [95\% CI: 50\%, 59\%]) $p=.401)$. For the scale measure, we found that participants rated the utilitarian response as more morally acceptable in the transfer-VOI condition $(M=4.19, S D=$ $1.80)$, as compared to those in the simple control condition $(M=3.94, S D=1.86 ; t(1387)=1.98$, $p=.048)$. However, there were no significant differences in participants' scale responses between the transfer-VOI condition and the anchoring control condition $(M=4.05, S D=1.78$; $t(1387)=1.08, p=.280$. (Figs. $1 \mathrm{G}, 2 \mathrm{~F}$.) These results establish a boundary condition on the effect 
of VOI reasoning. We note, however, that further training in VOI reasoning may enable people to transcend this boundary.

\section{Discussion}

Across multiple studies we show that veil-of-ignorance reasoning influences responses to moral dilemmas, encouraging responses that favor the greater good. These effects were observed in response to a classic philosophical dilemma, a bioethical dilemma, real-stakes decisions concerning charitable donations, and in judgments concerning policies for AVs. The effect on the AV dilemma was replicated in two pre-registered studies, which further showed that this effect cannot be explained by a generic tendency toward consistency and that it depends critically on assigning probabilities aligned with a principle of impartiality. Previous research indicates net disapproval of utilitarian regulation of AVs (17). Here, VOI reasoning shifts approval to as high as $83 \%$ (Fig. 1C, 1E-F). (We note that these findings address attitudes toward regulation, but not individual consumption.)

Arguably the most central debate in the field of moral psychology concerns whether and to what extent people's judgments are shaped by intuition as opposed to reason or deliberation $(10-12,16)$. There is ample evidence for the influence of intuition, while evidence for effectual moral reasoning is more limited (16). Beyond simple cost-benefit utilitarian reasoning (11-15), people's judgments are influenced by explicit encouragement to think rationally (23) and by simple debunking arguments (11). Performance on the Cognitive Reflection Test (24) is correlated with utilitarian judgment $(11,15,25)$, and exposure to this test can boost utilitarian judgment $(11,15)$, but this appears to simply shift the balance between intuitive responding and utilitarian reasoning, rather than eliciting a more complex form of reasoning. Closer to the 
present research is the use of joint (versus separate) evaluation, which induces participants to make a pair of judgments based on a common standard of evaluation (26).

Here we provide evidence for effectual moral reasoning in ordinary people that is arguably more complex than any previously documented. The VOI condition requires a kind of spontaneous "micro-philosophizing" to produce its effect, recapitulating the insights of Rawls and Harsanyi, who perceived an equivalence between self-interested decisions made from behind a veil of ignorance and justifiable moral decisions. Here, participants are not presented with an explicit argument. Instead, they're given the raw materials with which to construct and apply an argument of their own making. Participants in the VOI condition are not told that there is a normative relationship between the VOI exercise and the subsequent judgment, but many participants nevertheless perceive such a relationship. Without explicit instruction, they, perceive that a self-interested choice made from behind a veil of ignorance is an impartial choice, and therefore likely to be a morally good choice when the veil is absent. And, once again, this effect disappears when the probabilities are reversed, indicating that participants are sensitive to whether the VOI is fostering a kind of impartial thinking. We are not claiming, of course, that people engage in this kind of moral reasoning under ordinary circumstances. But these findings indicate that ordinary people can actively engage in a rather sophisticated kind of moral reasoning with no special training and minimal prompting.

We wish to note several limitations of the present findings. First, we do not claim that veil-of-ignorance reasoning must always promote utilitarian judgment. In particular, we are not attempting to resolve the debate between Rawls and Harsanyi over whether veil-of-ignorance reasoning favors a utilitarian principle over Rawls' "maximin" principle, as the dilemmas employed here do not distinguish between them. Second, we note that our studies aimed at ruling 
out competing explanations (Studies 4-5), as well as our study establishing limited generalization (Study 6), all used the AV policy case. Nevertheless, the most parsimonious interpretation of the evidence is that the VOI effect observed for the AV case is psychologically similar to those observed for other cases. Finally, we note that in Study 5 the proportion of utilitarian judgments in the standard AV case, following the reversed-VOI exercise, was relatively high (64\%), as compared to the stand-alone AV cases tested in Studies 2, 4, and $6(58 \%, 50 \%, 53 \%$, respectively). Thus, it is possible that component features of the VOI exercise, such as the engagement of probabilistic reasoning, may play some role in promoting subsequent utilitarian judgment. Alternatively, it could be that engaging in reversed-VOI reasoning is enough to prompt some participants to engage in standard VOI reasoning.

We wish to emphasize that these findings, by themselves, neither assume nor demonstrate that the effects of veil-of-ignorance reasoning are desirable. Nevertheless, these findings may have significant implications when combined with certain widely shared moral values (27). For those who regard promoting the greater good as an important moral goal, the present findings suggest a useful tool for encouraging people to make decisions that promote the greater good. Likewise, this approach may be of interest to those who value impartial procedures, independent of any commitment to maximizing aggregate well-being.

Veil-of-ignorance reasoning may be most useful when people are forced to make, and publicly justify, decisions involving difficult tradeoffs. Decisions that promote the greater good may involve emotionally aversive sacrifices and/or an unwillingness to allocate resources based on personal or group-based loyalties $(12,28)$. Observers tend to be highly suspicious of people who make utilitarian decisions of this kind (29). How, then, can decision-makers whose genuine aim is to promote the greater good advance policies that are so readily perceived as anti-social or 
disloyal? We suggest that veil-of-ignorance reasoning can help people-both decision-makers and observers - distinguish between policies that are truly anti-social or culpably disloyal from socially beneficial policies that are simply aversive. Emotionally uncomfortable tradeoffs may seem more acceptable if one can credibly say, "This is what I would want for myself if I did not know who I was going to be."

Where there is conflict_-either within or between people_-about important moral decisions, mechanisms that might promote agreement are worth considering. Veil-of-ignorance reasoning may be especially useful because it influences people's judgments without telling them how to think or what to value. Nor does it manipulate people through non-conscious influences or the restriction of information. Instead, it is Socratic. It openly and transparently asks people to consider their decisions from a different perspective, leaving it up to decision-makers to determine whether that perspective is valuable. Across a range of decisions, from bioethics to philanthropy to machine ethics, people seem to find this perspective illuminating.

\section{Materials and Methods}

The procedures and materials for all studies were reviewed and approved by the Harvard University Institutional Review Board. All participants provided informed consent. We have uploaded all study materials, preregistrations, raw data, and analyses code on Open Science Framework, accessible at the following link:

https://osf.io/6xyct/?view_only=13cafb7e7c654c1e84afcf6401716f7b.

All statistical analyses were conducted using R statistical software. For complete study materials, see Supporting Information.

Study 1. In both conditions, participants entered their Amazon Mechanical Turk (MTurk) IDs and completed an attention check. Participants who failed the attention check were excluded 
from analysis. In the control condition, participants responded to the standard version of the footbridge dilemma with a dichotomous choice ("Is it morally acceptable for you to push the second person on to the tracks in order to save the five workmen?") and a scale item ("To what extent is this action morally acceptable?").

In the VOI condition, participants first responded to a VOI version of the footbridge dilemma in which the participant is asked to imagine having an equal probability of being each of the six people affected by the decision. They then indicated what they would like the decisionmaker to do using a dichotomous choice ("Do you want the decision-maker to push or not push?") and a scale measure (To what extent do you want the decision-maker to push?). The VOI version of the footbridge dilemma was then followed by the standard version, as used in the control condition. Once again, in both conditions our primary dependent measures are responses to the standard footbridge dilemma.

In both conditions, after participants responded to the dilemma(s), they completed comprehension checks (one for each dilemma). Participants who failed the comprehension check(s) were excluded from analysis. In the VOI condition participants were asked about whether their responses to the standard case were influenced by the VOI exercise. All participants assessed their prior familiarity with the testing materials, supplied their age and gender, and were asked for general comments.

Study 2. Procedures followed those of Study 1, but using the hospital and AV dilemmas. Participants were assigned to the same condition for both dilemmas. In the VOI condition, participants were always presented with the VOI version of a dilemma immediately prior to the standard version of that dilemma. In both conditions, and in both stages of the VOI condition, the order of the dilemmas (AV vs. hospital) was counterbalanced. 
In the standard AV case, participants responded to the dichotomous measure ("Is it morally acceptable for a state law to require autonomous vehicles to swerve in such a situation to save the 9 pedestrians?") followed by the corresponding scale measure. In the standard hospital case, participants responded to the dichotomous measure ("Is it morally acceptable for you to take the patient at the hospital off oxygen?") followed by the scale measure.

In the VOI version of the AV dilemma, participants responded to a dichotomous measure ("Please respond from a purely self-interested perspective: Would you want to be in a state where the law requires autonomous vehicles to swerve in such a situation?") and a corresponding scale measure. Likewise, in the VOI hospital dilemma, participants responded to a dichotomous measure ("Please respond from a purely self-interested perspective: Do you want the ethics committee to take the patient off oxygen?') and a corresponding scale measure. In Study 1, we expected participants responding to the VOI dilemma to respond from a self-interested perspective when asked what they would want the decision-maker to do, as the decision-maker's choice would determine whether they would probably live or probably die. In Study 2 and all subsequent studies we explicitly instructed participants responding to VOI dilemmas to respond from a self-interested perspective. This change only affects the VOI exercise and not the standard moral dilemma used in the second phase of the VOI condition.

Study 3. Here participants chose between a more effective and less effective charitable donation. We presented all participants with descriptions of two real charities, although the charity names were not given. Donating \$200 to the Indian charity would fund cataract surgeries for 2 people in India. Each of the 2 people need surgery in one eye, and without the surgery, each will go permanently blind in one eye. Donating \$200 to the U.S. charity would contribute to funding surgery for a person living in the U.S. Here the recipient is going blind from an eye 
disease called pars planitis, and without the surgery, this person will go permanently blind in one eye. These charities were designated "Charity A" and "Charity B", with label/order counterbalanced.

The Indian charity is more effective because the same level of donation cures two people instead of one. More precisely, a donation to the U.S. charity is expected to contribute to the curing of a single person. However, for the purposes of assigning probabilities in the VOI version, we assumed that the single person would be cured as a result of the donation. This is a conservative assumption, since it increases the appeal of the U.S. charity, and our prediction is that considering the VOI version of the charity dilemma will make people less likely to choose the U.S. Charity in the subsequent decision. The two charities differ in other ways, most notably in the nationality of beneficiaries, but that is by design, as the most effective charities tend to benefit people in poorer nations, where funds go further.

For the real donation decision employed in both conditions, we told participants, "We will actually make a $\$ 200$ donation and one randomly chosen participant's decision will determine where the $\$ 200$ goes." They were then asked, “To which charity do you want to donate the \$200?" with the options, "I want to donate the $\$ 200$ to Charity A" or "I want to donate the \$200 to Charity B."

In the VOI condition, participants were first presented with a VOI version of the charity dilemma (hypothetical) which used this prompt: "Please respond from a purely self-interested perspective: To which charity do you want the decision-maker to donate the $\$ 200$ ?” There was no continuous measure in Study 3. Participants in the VOI condition then made their real charity decision, as in the control condition. We compared the real charity decisions between the VOI and control conditions. 
Study 4. Study 4 methods (pre-registered; \#6425 on AsPredicted.org) follow those used for the AV policy case in Study 2, but with no accompanying bioethical dilemma, and, critically, with the inclusion of a new anchoring control condition. In the anchoring control condition, participants first responded to the sculpture case (which reliably elicits utilitarian responses) before responding to the AV case.

Study 5. Study 5 methods (pre-registered; \#11473 on AsPredicted.org) follow that of Studies 2 and 4, using variants of the AV policy dilemma for both the VOI condition and the reversed-VOI control condition. As before, in the VOI condition, participants completed a VOI version of the AV dilemma, in which they imagined having a 9 in 10 chance of being one of the 9 people in the path of the AV and a 1 in 10 chance of being the single passenger in the AV. In the reversed-VOI version of the AV dilemma, they imagined having a 9 in 10 chance of being the single passenger in the AV and a 1 in 10 chance of being one of the nine pedestrians in the AV's path.

Study 6. In Study 6 (pre-registered, \#6157 on AsPredicted.org), as in Studies 4-5, all participants responded to the standard AV case. In the simple control condition, participants responded only to the standard AV case. In the transfer-VOI condition, participants completed the VOI hospital and VOI charity cases (respectively from Studies 2 and 3) before responding to the standard AV case. In the anchoring control condition, prior to the standard AV case, participants responded to the sculpture case from Study 4 and an additional case, the speedboat case, which also reliably elicits utilitarian judgments. Thus, as in Study 4, this control condition is intended to control for participants' making two affirmative utilitarian responses prior to responding to the standard AV case. We note that this control condition lacks the features introduced in Study 5 (which was run after Study 6). However, because our prediction 
concerning this control condition was not confirmed, the absence of these features does not affect our conclusions. In both the transfer-VOI condition and the anchoring control conditions, we counterbalanced the order of the two cases preceding the standard AV case. 


\section{References}

1. J. Rawls, A Theory of Justice. (Harvard, 1971).

2. N. Frohlich, J. A. Oppenheimer, C. L. Eavey, Laboratory results on Rawls's distributive justice. Brit. J. Poli. Sci. 17(1), 1-21 (1987).

3. T. Kameda, K. Inukai, S. Higuchi, A. Ogawa, H. Kim, T. Matsuda, M. Sakagami, Rawlsian maximin rule operates as a common cognitive anchor in distributive justice and risky decisions. Proc. Natl. Acad. Sci. U.S.A. 113(42), 11817-11822 (2016).

4. J. C. Harsanyi, Cardinal welfare, individualistic ethics, and interpersonal comparisons of utility. J Poli Econ 63(4), 309-321 (1955).

5. J. C. Harsanyi, Can the Maximin Principle Serve as a Basis for Morality? A Critique of John Rawls's Theory. Amer. Poli. Sci. Rev. 69(2), 594-606 (1975).

6. J. J. Thomson, The trolley problem. Yale Law J. 94,1395-1415 (1985).

7. J. D. Greene, R. B. Sommerville, L. E. Nystrom, J. M. Darley, J. D. Cohen, An fMRI investigation of emotional engagement in moral judgment. Sci. 293(5537), 2105-2108 (2001).

8. M. Koenigs, L. Young, R. Adolphs, D. Tranel, F. A. Cushman, M. Hauser, A. Damasio, Damage to the prefrontal cortex increases utilitarian moral judgements. Nat. 446(7138), 908911 (2007).

9. C. Hare, Should we wish well to all? Phil. Rev. 125(4), 251-272 (2016).

10. J. M. Paxton, J. D. Greene, Moral reasoning: Hints and allegations. Top. Cog. Sci., 2(3), 511527 (2010).

11. J. M. Paxton, L. Ungar, J. D. Greene, Reflection and reasoning in moral judgment. Cog. Sci. 36(1), 163-177 (2012). 
12. J. D. Greene, Moral Tribes: Emotion, Reason, and the Gap between Us and Them. (Penguin, 2013).

13. M. J. Crockett, Models of morality. Tre. Cog. Sci. 17(8), 363-366 (2013).

14. P. Conway, J. Goldstein-Greenwood, D. Polacek, J. D. Greene, Sacrificial utilitarian judgments do reflect concern for the greater good: Clarification via process dissociation and the judgments of philosophers. Cog. 179, 241-265 (2018).

15. I. Patil, M. M. Zucchelli, W. Kool, S. Campbell, F. Fornasier, M. Calò, G. Silani, M. Cikara, F. Cushman, Reasoning supports utilitarian resolutions to moral dilemmas across diverse measures PsyArXiv doi:10.31234/osf.io/q86vx (22 December, 2018).

16. J. Haidt, The Righteous Mind: Why Good People Are Divided by Politics and Religion. (Vintage 2012).

17. J. F. Bonnefon, A. Shariff, I. Rahwan, The social dilemma of autonomous vehicles Sci. 352(6293), 1573-1576 (2016).

18. D. Z. Morris, Mercedes-Benz's self-driving cars would choose passenger lives over bystanders. Fortune (15 October, 2016).

19. C. Robichaud, Liberty hospital simulation. Classroom exercise. (2015).

20. A. Tversky, D. Kahneman, Judgment under uncertainty: Heuristics and biases. Sci. 185(4157), 1124-1131 (1974).

21. J. Von Neumann, O. Morgenstern, Theory of Games and Economic Behavior (Commemorative Edition). (Princeton 2007).

22. A. D. Galinsky, G. B. Moskowitz, Perspective-taking: Decreasing stereotype expression, stereotype accessibility, and in-group favoritism. J. Pers. Soc. Psy. 78(4), 708-724 (2000). 
23. D. A. Pizarro, E. Uhlmann, P. Bloom, Causal deviance and the attribution of moral responsibility. J. Exp. Soc. Psy. 39(6), 653-660 (2003).

24. S. Frederick, Cognitive reflection and decision making. J. Econ. Pers. 19(4), 25-42 (2005).

25. J. M. Paxton, T. Bruni, J. D. Greene, Are 'counter-intuitive' deontological judgments really counter-intuitive? An empirical reply to Kahane et al. (2012). Soc. Cog. Aff. Neur. 9(9), 1368-1371 (2013).

26. M. H. Bazerman, G. F. Loewenstein, S. B. White, Reversals of preference in allocation decisions: Judging an alternative versus choosing among alternatives. Adm. Sci. Quar. 37(2), 220-240 (1992).

27. J. D. Greene, Beyond point-and-shoot morality: Why cognitive (neuro)science matters for ethics. Ethics 124(4), 695-726 (2014).

28. P. Bloom, Against Empathy: The Case for Rational Compassion. (Ecco, 2016).

29. J. A. C. Everett, N. S. Faber, J. Savulescu, M. J. Crockett, The costs of being consequentialist: Social inference from instrumental harm and impartial beneficence. $J$. Exp. Soc. Psy. 79, 200-216 (2018).

30. M. J. Sandel, Justice: What's the Right Thing To Do?. (Macmillan, 2010).

31. F. H. Knight, Risk, Uncertainty and Profit. (Hart, Schaffner \& Marx, 1921). 


\section{Figure 1}

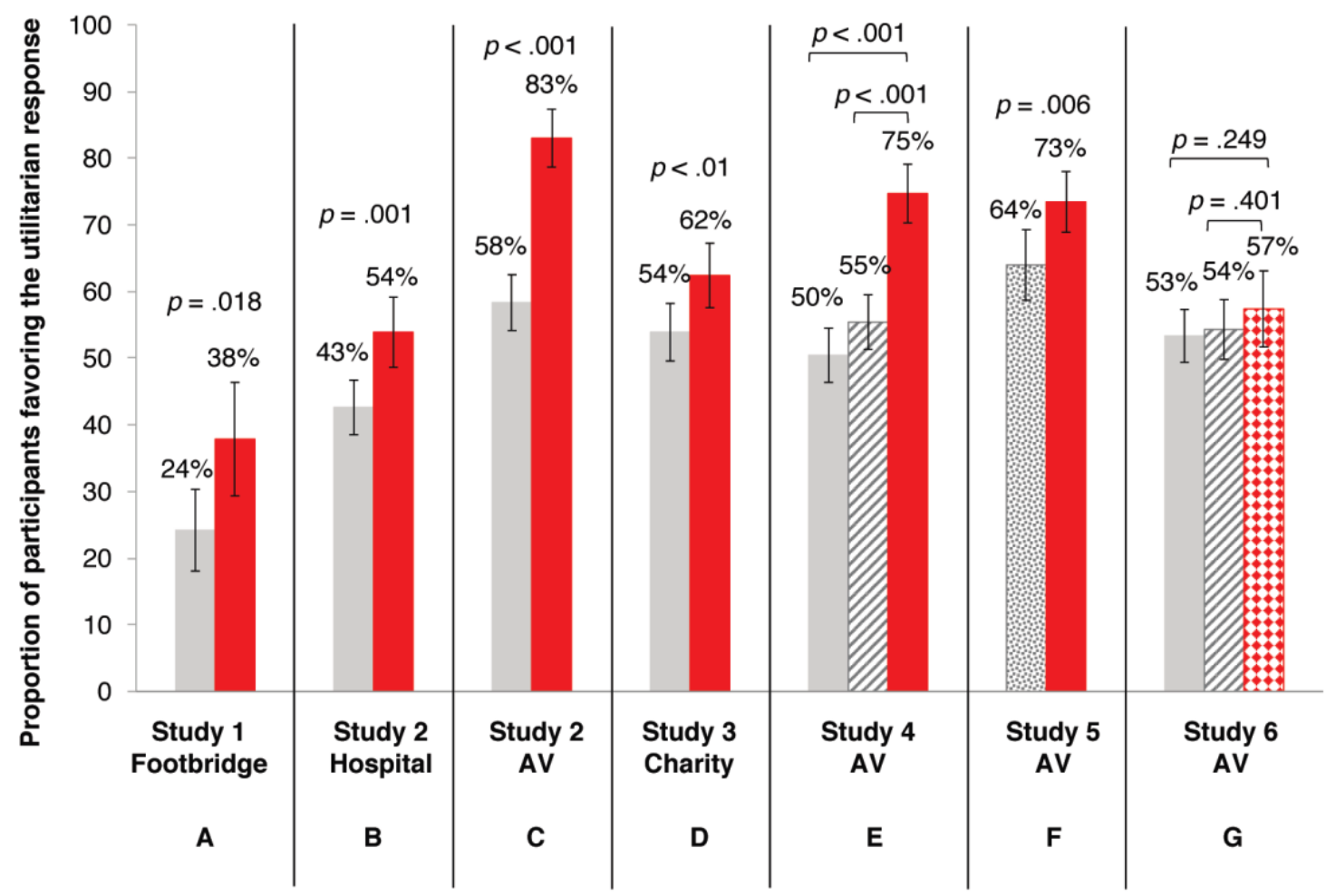

VOI Transfer VOI Simple Control $\mathbb{Z}$ Anchoring Control $\quad$ Reversed-VOI Control

Dichotomous responses for all studies $(N=5,785)$. $p$-values from logistic regression. Error bars indicate 95\% CI. (A) Study 1 footbridge case; (B) Study 2 hospital case; (C) Study 2 AV case; (D) Study 3 charity case; $(E)$ Study 4 AV case; $(F)$ Study 5 AV case; $(G)$ Study 6 AV case. 
Figure 2

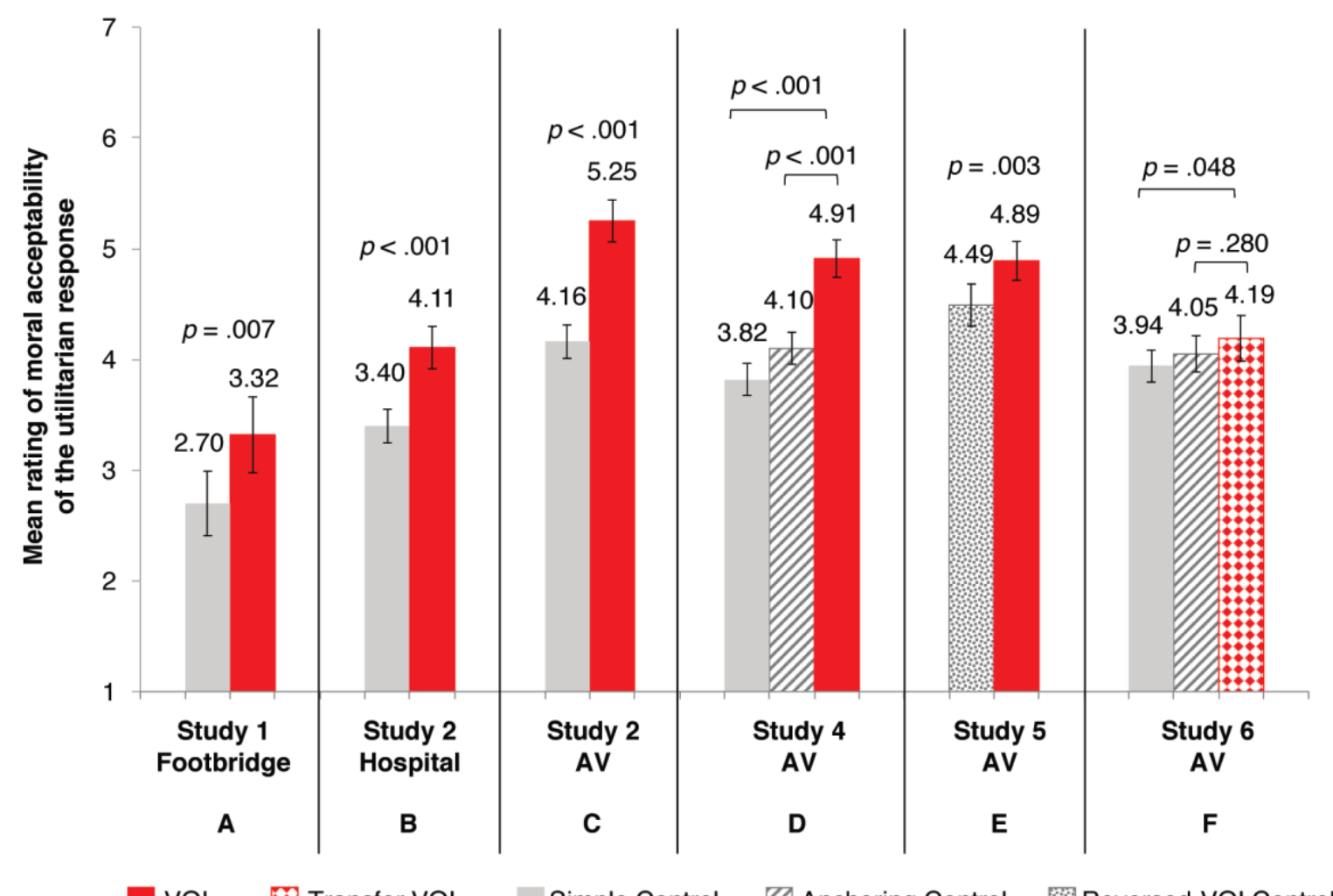

VOI Transfer VOI Simple Control $\square$ Anchoring Control 国 Reversed-VOI Control

Scale responses for Studies 1-2, 4-6 $(N=4857)$. $p$-values from linear regression. Error bars indicate $95 \%$ CI. (A) Study 1 footbridge case; (B) Study 2 hospital case; (C) Study 2 AV case; (D) Study 4 AV case; (E) Study 5 AV case; (F) Study 6 AV case. 